\title{
Deuteron and proton beams polarimetry at internal target at JINR Nuclotron
}

\author{
Vladimir Ladygin* \\ Joint Institute for Nuclear Research, Dubna, Russia \\ E-mail: vladyginejinr.ry
}

V.P. Ladygin ${ }^{1}$, A.V. Averyanov ${ }^{1}$, S.N. Bazylev ${ }^{1}$, A.S. Belov ${ }^{2}$, E.V. Chernykh ${ }^{1}$, D. Enache ${ }^{3}$, V.V. Fimushkin ${ }^{1}$, Yu.V. Gurchin ${ }^{1}$, A.Yu. Isupov ${ }^{1}$, M. Janek ${ }^{4}$, J.-T. Karachuk ${ }^{1,3}$, A.N. Khrenov ${ }^{1}$, D.O. Krivenkov ${ }^{1}$, P.K. Kurilkin ${ }^{1}$, A.N. Livanov ${ }^{1}$, S.M. Piyadin ${ }^{1}$, S.G. Reznikov ${ }^{1}$, Ya.T. Skhomenko ${ }^{1,5}$, I.V. Slepnev ${ }^{1}$, V.M. Slepnev ${ }^{1}$, A.V. Shutov ${ }^{1}$, A.A. Terekhin ${ }^{1}$, A.V. Tishevsky ${ }^{1}$, I.E. Vnukov ${ }^{5}$, I.S. Volkov ${ }^{5}$ (DSS Collaboration)

${ }^{1}$ Joint Institute for Nuclear Research, Dubna, Russian Federation

2 Institute of Nuclear Physics, Moscow, Russian Federation

${ }^{3}$ National Institute for $R \& D$ in Electrical Engineering ICPE-CA, Bukharest, Romania

${ }^{4}$ Physics Department, University of Žilina, Žilina, Slovak Republic

${ }^{5}$ Belgorod State National Research University, Belgorod, Russian Federation

\begin{abstract}
The spin program at SPD NICA as well as the experiments at Nuclotron require high intensity polarized proton and deuteron beams with high value of the polarization. The upgraded deuteron beam polarimeter at internal target at Nuclotron has been used to obtain the vector and tensor polarization for 6 different spin modes of new polarized source of ions. The values of the beam polarization was about $65-75 \%$ from their ideal values. The longterm stability of the deuteron beam polarization was demonstrated.

The polarization of the firstly accelerated at Nuclotron proton beam has been measured at 500 $\mathrm{MeV}$ using quasi-elastic proton-proton scattering using internal target polarimeter. The obtained value of the vertical polarization was about about 35\%. Further perspectives of the beam polarimetry development for NICA is discussed.
\end{abstract}

23rd International Spin Physics Symposium - SPIN2018 -

10-14 September, 2018

Ferrara, Italy

\footnotetext{
* Speaker.
} 


\section{Introduction}

The study of the spin structure of two-nucleon and three-nucleon short-range correlations via the measurements of the polarization observables in the deuteron induced reactions is main goal of the DSS project at Nuclotron [1, 2, 3]. The high precision polarimetry of the deuteron and proton beams in a wide energy range is important for these investigations.

On the other hand, the availability of the polarized proton beam is required by the spin program at NICA [ [ ]. The main part of the physics program with SPD detector consists of the measurements of asymmetries in the lepton pair (Drell-Yan) production in collisions of non-polarized, longitudinally and transversally polarized protons and deuterons beams. These measurements can provide an access to all leading twist collinear and Transverse-Momentum Dependent ditsribution functions of quarks and anti-quarks in nucleons. The measurements of asymmetries in production of $J / \Psi$ and direct photons, which supply complimentary information on the nucleon structure, will be performed simultaneously with Drell-Yan data using dedicated triggers. The set of these measurements permits to tests the quark-parton model of nucleons at the QCD twist-2 level with minimal systematic errors. This program can be extended by the measurements of the single and double spin asymmetries in the pion, kaon, proton inclusive production, vector mesons and hyperon production in polarized proton-proton collisions etc. Moreover, polarized proton beam is needed for the spin studies with fixed target, namely, measurements of spin observables proton-proton, proton-neutron and proton-deuteron elastic scattering, investigation of the spin structure of the short-range nucleon correlations and three nucleon forces [5]. The realization of this program requires good knowledge of the proton beam polarization.

The goal of the present article is to report new results on the measurements of the vector and tensor components of the polarized deuteron beam using upgraded polarimeter based on the asymmetry measurements in $d p$ - elastic scattering at $270 \mathrm{MeV}$ [6] at the Internal Target Station (ITS) [7] at Nuclotron. These measurements were performed within the DSS experiment on the study of the vector $A_{y}$, tensor $A_{y y}$ and $A_{x x}$ analyzing powers in $d p$ - elastic scattering at large transverse momenta [8, 9] using polarized deuteron beam from new source of polarized ions (SPI) [10]. Also first results of the proton beam polarization measurements performed at the Nuclotron ITS are reported.

\section{Polarimeter at ITS}

Efficient polarimetry can be achieved even at relatively low beam intensity using a thin solid target inside the inner ring of the accelerator. The luminosity can be increased significantly due to multiple beam passage through the interaction point and the use of a correctly configured internal target trajectory. Therefore, the internal beam polarimeter with a very thin target may have approximately the same efficiency as the extracted beams polarimeters. The polarimeter based on the use of $d p$ - elastic scattering at large angles $\left(\theta_{\mathrm{cm}} \geq 60^{\circ}\right)$ at $270 \mathrm{MeV}$, 6], where precise data on analyzing powers [11, 12, 13] exist, has been developed at ITS at Nuclotron [7]. The accuracy of the determination of the deuteron beam polarization achieved with this method is better than $2 \%$ because of the values of the analyzing powers were obtained for the polarized deuteron beam, which absolute polarization had been calibrated via the ${ }^{12} \mathrm{C}(d, \alpha){ }^{10} \mathrm{~B}^{*}\left[2^{+}\right]$reaction[13]. 
The ITS setup is well suited for study of the energy dependence of polarization observables for the deuteron-proton elastic scattering and deuteron breakup reaction with the detection of two protons at large scattering angles. For these purposes the $\mathrm{CH}_{2}$-target of $10 \mu \mathrm{m}$ thick is used for the measurements. The yield from carbon content of the $\mathrm{CH}_{2}$-target is estimated in separate measurements using several twisted $8 \mu \mathrm{m}$ carbon wires. Deuteron beam polarimeter [6] is placed in the Nuclotron ring. It consists of a spherical scattering chamber and targets change system that can be set six different targets. A detector support with 39 mounted plastic scintillation counters is placed downstream the ITS spherical chamber. Each plastic scintillation counter was coupled to a photo-multiplier tube Hamamatsu H7416MOD. Eight proton detectors were installed for left arm, as well as for right and up, but due to space limitation - only four for down. The angular span of one proton detector was $2^{\circ}$ in the laboratory system, which corresponds to $\sim 4^{\circ}$ in the cms. Three deuteron detectors are placed at scattering angles of deuterons coinciding kinematically with the protons. Only one deuteron detector can cover the solid angle corresponding to four proton detectors placed down. The scattered deuterons and recoil protons at $270 \mathrm{MeV}$ were detected in kinematic coincidence over the cms angular range of $65^{\circ}-135^{\circ}$ at eight different angles, defined by the positions of the proton detectors. The beam intensity monitoring is done from the detection of $p p$ - quasielastic scattering at $90^{\circ}$ in $\mathrm{cms}$ by the scintillation counters placed in the horizontal plane by the additional pair of detectors.

The VME (Versa Module Eurocard) based data acquisition system with new software [14] is used for the data taking from scintillation detectors. The signals from the detectors are fed in 16channel TQDC-16 charge-time-digital [15] converters via commutator bar. TQDC-16 module allows one to measure the amplitude and time appearance of the signal simultaneously. The hardware of the DSS VME system consists of 4 TQDC-16 modules, trigger modules TTCM or TMWR and U40 and VME controller [15]. There is a possibility to tune the first-level trigger using firmware logic of trigger and TQDC-16 modules. Newly developed multichannel high-voltage power supply system based on Wiener MPod [16] is used to provide the power for about 70 scintillation detectors equipped with Hamamatsu photomultipliers.

The setup has been also adopted for the measurements of the proton beam polarization at 500 $\mathrm{MeV}$ using $p p$ - quasielastic scattering.

\section{Results on the deuteron beam polarization measurement}

The deuteron beam polarization measurements were performed using ITS at Nuclotron [诃 with new control and data acquisition system [17]. New source of polarized ions [10] has been used to provide polarized deuteron beam. In the current experiment the spin modes with the maximal ideal values of $\left(P_{z}, P_{z z}\right)=(0,0),(-1 / 3,+1)$ and $(-1 / 3,+1)$ were used. The deuteron beam polarization has been measured at $270 \mathrm{MeV}$ [6]. The DSS data taking was separated on 3 parts: November 2016, December 2016 and February 2017. The deuteron beam polarization measurements were performed at $270 \mathrm{MeV}$ before and after each energy studied in the range of 400-1800 MeV [18].

The software for data analysis was in $\mathrm{C}++$ developed using the ROOT package. It consists of several classes and methods. This software can read ROOT representation of the experimental data, graphical windows, save vector and ruster results, graphics and cuts. 


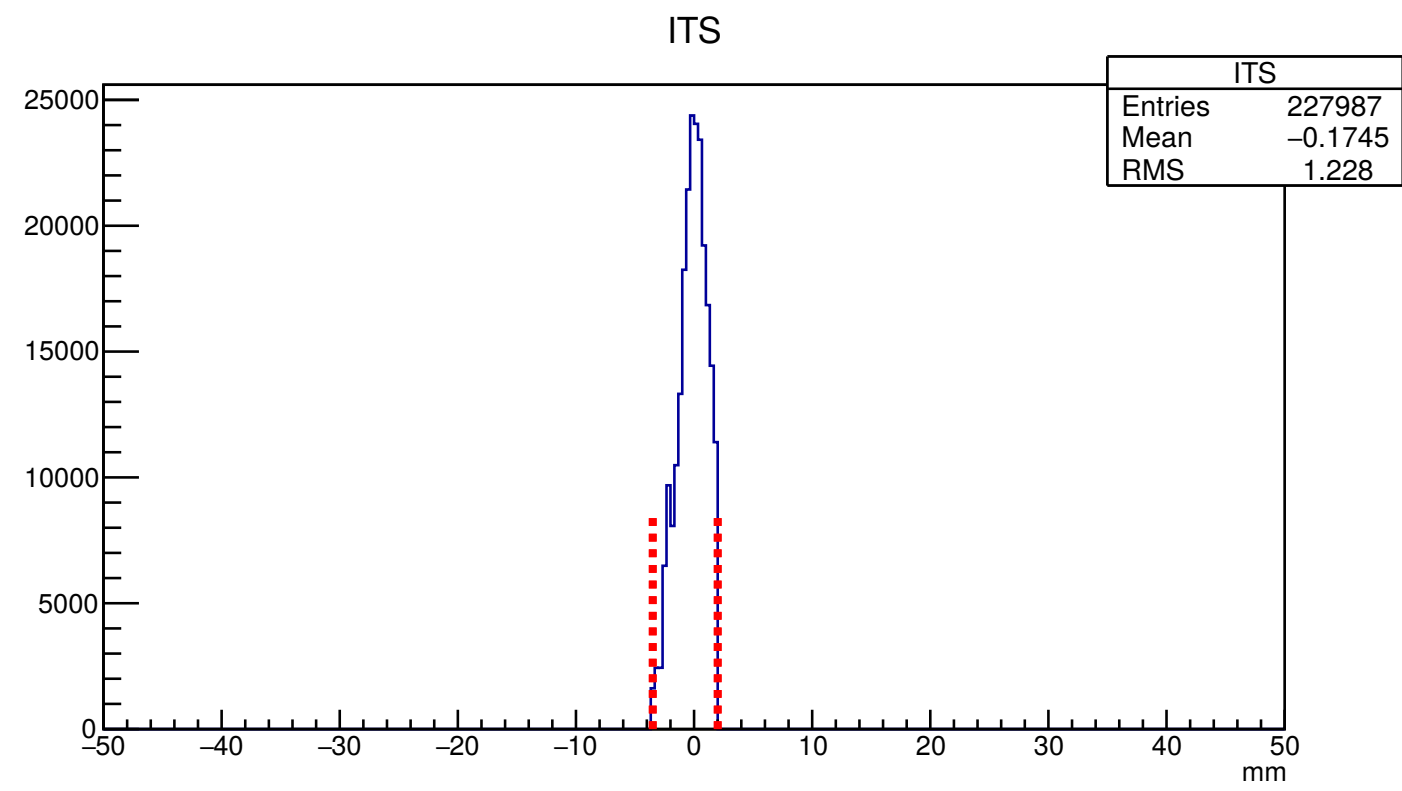

Figure 1: The internal target position monitor selection window.

The $d p$ - elastic scattering events at $270 \mathrm{MeV}$ were selected using information on the interaction point (target position monitor), correlation of the energy losses and time-of-flight difference for deuteron and proton detectors. Firstly, data are selected using the information on the internal target position monitor (see Fig. 1). This criterion reject all detector signals which come when target is outside of the beam. The selection procedure requires the use of only one graphical window for all pairs of detectors. The next step is the using of the graphical windows for time-of-flight difference (see upper panels in Fig. 2). One dimensional histograms obtained for polyethylene target has a sharp hydrogen peak and wide carbon background. Final step is the cut application for the correlation of the energy losses (see bottom panels in Fig. 2), where we can see 2D histograms with clean hydrogen signal. The measurements were performed using $\mathrm{CH}_{2}$ target only. The carbon contamination was measured to be less than $\sim 0.5 \%$ after using all graphical windows. Therefore, the subtraction of the carbon content was not applied.

The vector and tensor polarizations were measured seven, six and four times in the parts at November-2016, December-2016 and February-2017, respectively. The values have small statistical and systematics errors. They are rather stable within each part of the experiment. The exception is the December-2016 part, when the physics program was separated on two parts by the tuning of the SPI for pure tensor modes $\left(P_{z}, P_{z z}\right)=(0,-2)$ and $(0,+1)$ during 8 hours. This is the reason why December-2016 part is divided by two sets of the measurements. The polarization values were approximated by the constants for all four sets of the data. The results of the measurements and approximation are presented in Fig. 3. All the results are within two standard deviations from these constants. One can see that the beam polarization values are quite stable within more than 200 hours of the SPI operation. On the other hand, SPI demonstrates good reproducibility of the polarization values for different sets of the data after long interruptions. The values of the beam polarization for different spin have been obtained as weighted averages for 8 scattering angles for 

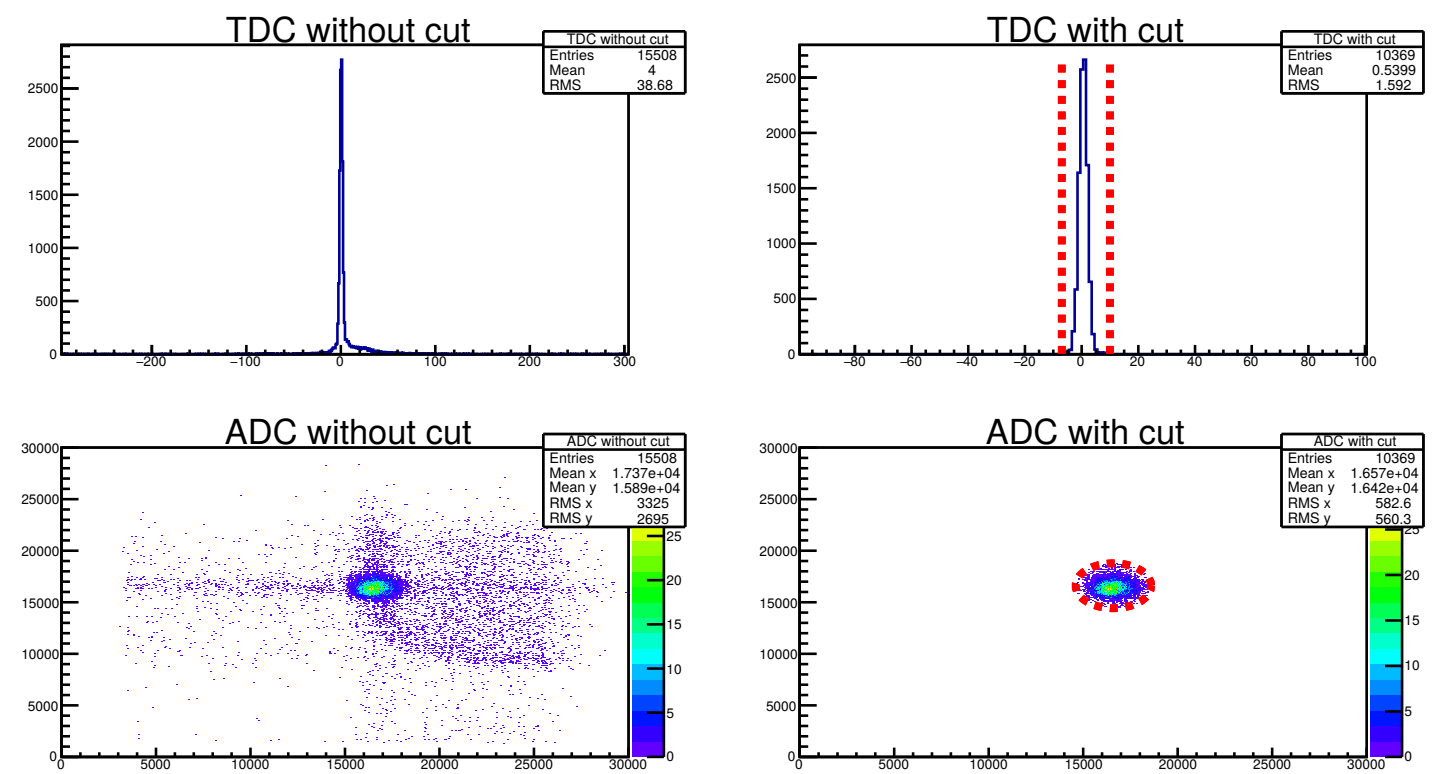

Figure 2: The selection of dp-elastic scattering events using time-of-flight difference and energy losses correlation.
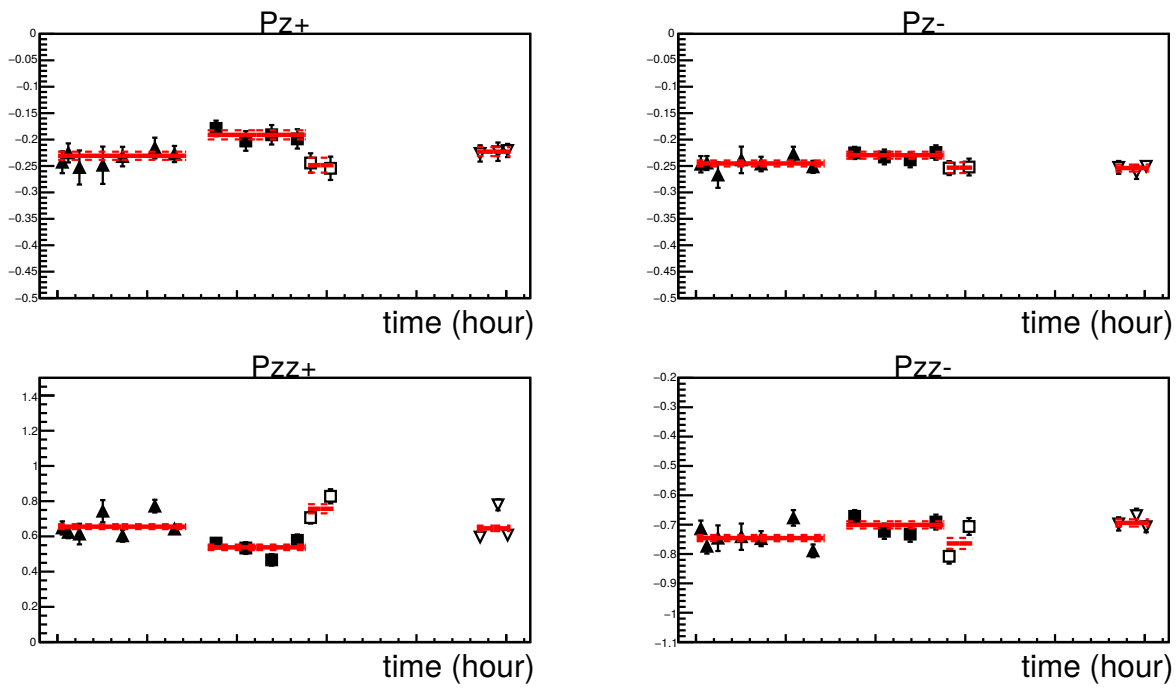

Figure 3: Polarizations values during the runs in 2016 and 2017 yy.

$d p$ - elastic scattering in the horizontal plane only. The typical values of the beam polarization were $\sim 65-75 \%$ from the ideal values.

The typical values of the vector and tensor components of the beam polarization for the spin modes $(-1 / 3,-1)$ and $(-1 / 3,+1)$ are given in table 1. The polarimeter [6] has been used also to tune the SPI operation for pure tensor spin modes $(0,-2)$ and $(0,+1)$, for pure vector spin mode $(-2 / 3,0)$ and for the spin mode $(-1,+1)$ with both vector and tensor components. The preliminary results are also presented in table 1. One can see, that the typical values of the beam 
Table 1: The vector and tensor polarizations for different spin modes

\begin{tabular}{lllll}
\hline Spin mode $\left(P_{z}, P_{z z}\right)$ & $P_{z}$ & $d P_{z}$ & $P_{z z}$ & $d P_{z z}$ \\
\hline$(-1 / 3,+1)$ & -0.272 & 0.019 & 0.733 & 0.035 \\
\hline$(-1 / 3,-1)$ & -0.272 & 0.014 & -0.793 & 0.026 \\
\hline$(0,+1)$ & 0.040 & 0.023 & 0.725 & 0.042 \\
\hline$(0,-2)$ & 0.042 & 0.013 & -1.478 & 0.030 \\
\hline$(-1,+1)$ & -0.648 & 0.016 & 0.628 & 0.027 \\
\hline$(-2 / 3,0)$ & 0.468 & 0.013 & 0.039 & 0.021 \\
\hline
\end{tabular}

polarization were $\sim 65-75 \%$ from the ideal values for all 6 spin modes of SPI.

\section{Results of the proton beam polarization measurement}

The classical method to measure the proton beam polarization at intermediate and high energies is the use of the left-right $p p$ - elastic or quasi-elastic scattering (see, for instance, [19] and references therein). The maximal value of the analyzing power at the energies below $1000 \mathrm{MeV}$ is close to $\sim 40^{\circ}$ in $\mathrm{cms}$ [20], that corresponds roughly $14-15^{\circ}$ in the laboratory. Unfortunately, this angle is inaccessible due to design of ITS and detector support.

This method has been modified to increase the polarimeter figure of merit. For this purpose the measurements of the proton beam polarization using several pairs of the detectors placed in the kinematic coincidences corresponding to $p p$ - elastic scattering in the horizontal plane (orbit plane of Nuclotron) have been proposed. The similar method has been used at COSY using EDDA setup [21, 22]. The feasibility of the proposed method at ITS has been checked in November 2016 run at Nuclotron using polarized deuteron beam with the energy of $500 \mathrm{MeV} /$ nucleon.

New SPI [10] has been used to provide polarized deuteron beam. In the current experiment the spin modes with the maximal ideal values of $\left(P_{z}, P_{z z}\right)=(0,0),(-1 / 3,+1)$ and $(-1 / 3,+1)$ were used. The deuteron beam polarization has been measured at $270 \mathrm{MeV}$ [6]. The $d p$ - elastic scattering events at $270 \mathrm{MeV}$ were selected using correlation of the energy losses and time-of-flight difference for deuteron and proton detectors. The values of the beam polarization for different spin have been obtained as weighted averages for 8 scattering angles for $d p$ - elastic scattering in the horizontal plane only. They were measured as $\left(P_{z}, P_{z z}\right)=(-0.232 \pm 0.018,+0.595 \pm 0.013)$ and $(-0.243 \pm 0.013,-$ $0.736 \pm 0.011)$ for spin modes "2-6" and "3-5", respectively.

After deuteron beam polarization measurements at $270 \mathrm{MeV}$, the beam has been accelerated up to $1000 \mathrm{MeV}$ (or $500 \mathrm{MeV} /$ nucleon). Eight pairs of the scintillation detectors were positioned in the horizontal plane covering angular range $55^{\circ}-125^{\circ}$ in the $\mathrm{cms}$ for $p p$-elastic scattering at 500 $\mathrm{MeV}$ on the left. Since analyzing power is antisymmetric with respect to $\theta^{*}=90^{\circ}$, the scattering in the backward hemisphere on the left at the angle $\pi-\theta$ can be considered as the scattering in the forward hemisphere on the right at the angle $\theta[23]$.

The main part of the measurements were performed using $\mathrm{CH}_{2}$ target. Carbon target was used to estimate the background. The $p p$ - quasielastic scattering events were selected using the energy 
losses and time-of-flight difference for two proton detectors placed in the kinematic coincidences. The normalized numbers of $p p$-elastic scattering events for each spin mode were used to calculate the values of the analyzing power of $p p$ - elastic scattering at $500 \mathrm{MeV}$. The obtained results were found to be in good agreement with the SP07 solution of SAID PWA [20], that proved the feasibility of the proposed method.

The unpolarized and polarized proton beam provided by SPI [10] has been accelerated in March 2017 run up to $500 \mathrm{MeV}$. The typical intensity of the beam was $\sim 1.5 \cdot 10^{8}$ ppp and $\sim 2-3 \cdot 10^{7}$ ppp for unpolarized and polarized cases, respectively. SPI provided proton beam polarization using WFT $1 \rightarrow 3$ with ideal value of the polarization $P=-1$. The polarization of the proton beam has been obtained using the data from eight pairs of the detectors placed in the kinematic coincidences. The values of the analyzing power for $p p$ - elastic scattering were taken from SAID PWA [20].

The weighted average values of the proton beam polarization were found as $0.017 \pm 0.021$ and $-0.354 \pm 0.022$ for unpolarized and polarized cases, respectively.

The availability of the polarized proton beam at Nuclotron allows to extend the DSS physics program at ITS [5], namely, to perform the experiments on the measurements of the nucleon analyzing power $A_{y}^{p}$ in $p d$ - elastic scattering at $135-1000 \mathrm{MeV}$ and in $p d$-nonmesonic breakup at the energies between $135-250 \mathrm{MeV}$ for different kinematic configurations etc.

\section{Conclusions}

- The upgraded version of the deuteron beam polarimeter [6] has been used to obtain the vector and tensor polarizations using $d p$ - elastic scattering at $270 \mathrm{MeV}$ during 2016/2017 Nuclotron runs.

- The time stability of the polarization values has been demonstrated for the spin modes of new SPI [10] $(-1 / 3,+1)$ and $(-1 / 3,-1)$.

- The polarimeter has been used for tuning of the ion source parameters for 6 different spin modes. The polarization values were $70-75 \%$ from the ideal ones.

- The current polarimeter has been also used to measure the proton beam polarization at 500 $\mathrm{MeV}$ using $p p$ - quasielastic scattering. The obtained value of the vertical proton polarization is $-0.354 \pm 0.022$.

The authors thank the Nuclotron staff for providing good conditions of the experiment. They thank A.V Butenko, A.D. Kovalenko, A.O. Sidorin for providing of the polarized proton beam and V.B. Shutov for the tune of the SPI [10]. They express the gratitude to V.I. Maximenkova for the help during the preparation of the detection system. They thank Yu.N. Filatov, A.M. Kondratenko and M.A. Kondratenko for useful discussions. The work has been supported in part by the RFBR under grant $N^{0} 16-02-00203 \mathrm{a}$, by the Ministry of Education, Science, Research, and Sport of the Slovak Republic (VEGA Grant No. 1/0113/18), by JINR- Slovak Republic and JINR-Romania scientific cooperation programs in 2016-2018. 


\section{References}

[1] V.P. Ladygin et al., Spin physics in few body systems at Nuclotron, Phys.Part.Nucl. 45 (2014) 327.

[2] V.P. Ladygin et al., Few-body Studies at Nuclotron-JINR, Few Body Syst. 55 (2014) 709.

[3] M. Janek et al., Investigation of the dp breakup and dp elastic reactions at intermediate energies at Nuclotron, Few Body Syst. 58 (2017) 40.

[4] http://nica.jinr.ru

[5] V.P. Ladygin et al., The spin studies in few-body systems at Nuclotron Int. J. Mod. Phys. Conf. Ser. 40 (2016) 1660074.

[6] P.K. Kurilkin et al., The $270 \mathrm{MeV}$ deuteron beam polarimeter at the Nuclotron Internal Target Station, Nucl.Instr.Meth. in Phys.Res. A642 (2011) 45.

[7] A.I. Malakhov et al., Potentialities of the internal target station at the Nuclotron, Nucl.Instrum.Meth. in Phys.Res. A440 (2000) 320.

[8] P.K. Kurilkin et al., Measurement of the vector and tensor analyzing powers for dp-elastic scattering at $880 \mathrm{MeV}$, Phys.Lett. B715 (2012) 61.

[9] P.K. Kurilkin et al., Investigation of the angular dependence of the analyzing powers in the deuteron-proton elastic scattering at the nuclotron, Phys.Part.Nucl.Lett. 8 (2011) 1081.

[10] V.V. Fimushkin et al., Development of polarized ion source for the JINR accelerator complex, J.Phys.Conf.Ser. 678 (2016) 012058;

A.S. Belov et al., Source of polarized ions for the JINR accelerator complex, J.Phys.Conf.Ser. 938 (2017) 012017.

[11] K. Sekiguchi et al., Complete set of precise deuteron analyzing powers at intermediate energies: comparison with modern nuclear force predictions, Phys.Rev. C65 (2002) 034003.

[12] K. Sekiguchi et al., Polarization transfer measurement for ${ }^{1} H(\vec{d}, \vec{p})^{2} H$ elastic scattering at 135-MeV/u and three nucleon force effects, Phys.Rev. C70 (2004) 014001.

[13] K. Suda et al., Absolute calibration of the deuteron beam polarization at intermediate energies via the ${ }^{12} \mathrm{C}(d, \alpha){ }^{10} \mathrm{~B}^{*}\left[2^{+}\right]$reaction, Nucl.Instr.Meth. in Phys.Res. A572 (2007) 745.

[14] A.Yu. Isupov, Online polarimetry of the Nuclotron internal deuteron and proton beams, J.Phys.Conf.Ser. 938 (2017) 012019.

[15] http://afi.jinr.ru

[16] Ya.T. Skhomenko et al., Software development for multi-channel LV/HV supply system based on Wiener MPod, BSU Sci.Bull. Math.\& Phys. 234 (2016) 126.

[17] A.Yu. Isupov, V.A. Krasnov, V.P. Ladygin, S.M. Piyadin, S.G. Reznikov, The Nuclotron internal target control and data acquisition system, Nucl.Instrum.Meth. in Phys.Res. A698 (2013) 127.

[18] V.P. Ladygin et al., Spin studies of the short-range correlations at Nuclotron, PoS SPIN2018 (2018) 023.

[19] L.S. Azhgirey et al., Intermediate-energy polarimeter for the measurement of the deuteron and proton beam polarization at the JINR synchrophasotron, Nucl.Instrum.Meth. in Phys.Res. A497 (2003) 340.

[20] http://gwdac.phys.gwu.edu 
[21] M. Altmeier et al., Excitation functions of the analyzing power in elastic proton-proton scattering from 0.45 to $2.5 \mathrm{GeV}$, Eur.Phys.J. A23 (2005) 351.

[22] F. Bauer et al.,Excitation functions of spin correlation parameters $A_{N N}, A_{S S}$ and $A_{S L}$ in elastic $\vec{p} \vec{p}$ scattering between $0.45 \mathrm{GeV}$ and $2.5 \mathrm{GeV}$, Phys.Rev. C71 (2005) 054002.

[23] C. Lechanoine-LeLuc and F. Lehar, Nucleon- nucleon elastic scattering and total cross sections, Rev.Mod.Phys. 65 (1993) 47. 